\title{
Experiments on Morphological Reinflection: CoNLL-2017 Shared Task
}

\author{
Akhilesh Sudhakar \\ BITS, Pilani, India \\ akhileshs.s4@gmail.com
}

\author{
Anil Kumar Singh \\ IIT (BHU), Varanasi, India \\ nlprnd@gmail.com
}

\begin{abstract}
We present two systems for the task of morphological inflection, i.e., finding a target morphological form, given a lemma and a set of target tags. Both are trained on datasets of three sizes: low, medium and high. The first uses a simple Long Short-Term Memory (LSTM) for lowsized dataset, while it uses an LSTMbased encoder-decoder based model for the medium and high sized datasets. The second uses a simple Gated Recurrent Unit (GRU) for low-sized data, while it uses a combination of simple LSTMs, simple GRUs, stacked GRUs and encoderdecoder models, depending on the language, for medium-sized data. Though the systems are not very complex, they give accuracies above baseline accuracies on high-sized datasets, around baseline accuracies for medium-sized datasets but mostly accuracies lower than baseline for low-sized datasets.
\end{abstract}

\section{Introduction}

The CoNLL-SIGMOPRHON 2017 shared task Cotterell et al. (2017) consists of two subtasks out of which we participate only in the first subtask, which involves generating a target inflected form from a given lemma with its part-of-speech. For instance, the word writing is the present continuous inflected form of the lemma write. The models were trained on three differently-sized datasets. The low-sized datasets had around 100 training samples, the medium-sized datasets had around 1000 training samples and the high-sized datasets had around 10000 samples for most languages. Datasets were provided for a total of 52 languages.

\section{Background}

Prior to neural network based approaches to morphological reinflection, most systems used a 3step approach to solve the problem: 1) String alignment between the lemma and the target (morphologically transformed form), 2) Rule extraction from spans of the aligned strings and 3) Rule application to previously unseen lemmas to transform them. Durrett and DeNero (2013) and Ahlberg et al. (2014; 2015) used the above approaches, with each of them using different string alignment algorithms and different models to extract rules from these alignment tables. However, in these kinds of systems, the types of rules to be generated must be specified, which should also be engineered to take into account language-specific transformational behaviour.

Faruqui et al. (2016) proposed a neural network based system which abstracts away the above steps by modeling the problem as one of generating a character sequence, character-by-character. Akin to machine translation systems, this system uses an encoder-decoder LSTM model as proposed by Hochreiter and Schmidhuber (1997). The encoder is a bidirectional LSTM, while the decoder LSTM feeds into a softmax layer for every character position in the target string. A beam search is used to create many output sequences and the best one is chosen based on predicted scores from the softmax layer. This model takes into account the fact that the target and the root word are similar, except for the parts that have been changed due to inflection, by feeding the root word directly to the decoder as well. A separate neural net is trained for every language.

\section{System Description}

We have modeled our system based on the system proposed by Faruqui et al. (2016), as described 
in the previous section. However we have made some modifications to the above system, to account for the three different sizes of datasets and to account for the behaviour of morphological transformations of independent languages. We submitted two submissions for the shared task, each of which we describe in the following sections.

In all the models, some structural and hyperparametrical features remain the same. The characters in the root word are represented using character indices, while the morphological features of the target word are represented using binary vectors. Each character of the root word is then embedded as a character embedding of dimension 64 , to form the root word embedding. If an encoder is used, it is bidirectional and the the input word embeddings feed into it. The output of the encoder (if any), concatenated with the root word embedding, feeds into the decoder. All recurrent units have hidden layer dimensions of 256, meaning that they transform the input to a vector of dimension 256. Over the decoder layer is a softmax layer that is used to predict the character that must occur at each character position of the target word. In order to maintain a constant word length, we use paddings of ' 0 ' characters. All models use categorical cross-entropy as the loss function and the Adam optimizer as reported by Kingma and $\mathrm{Ba}$ (2014) for optimization.

\subsection{First Submission}

\subsubsection{Low-sized Dataset}

For training the model on the low-sized dataset, we did not use any encoder and we used a simple LSTM with a single layer as the recurrent unit (Figure 1).

\subsubsection{Medium-sized Dataset}

For training the model on the medium-sized dataset, we used a bidirectional LSTM as the encoder and a simple LSTM with a single layer as the decoder (Figure 2).

\subsubsection{High-sized Dataset}

For training the model on the high-sized dataset, we used a bidirectional LSTM as the encoder and a simple LSTM with a single layer as the decoder (Figure 2).

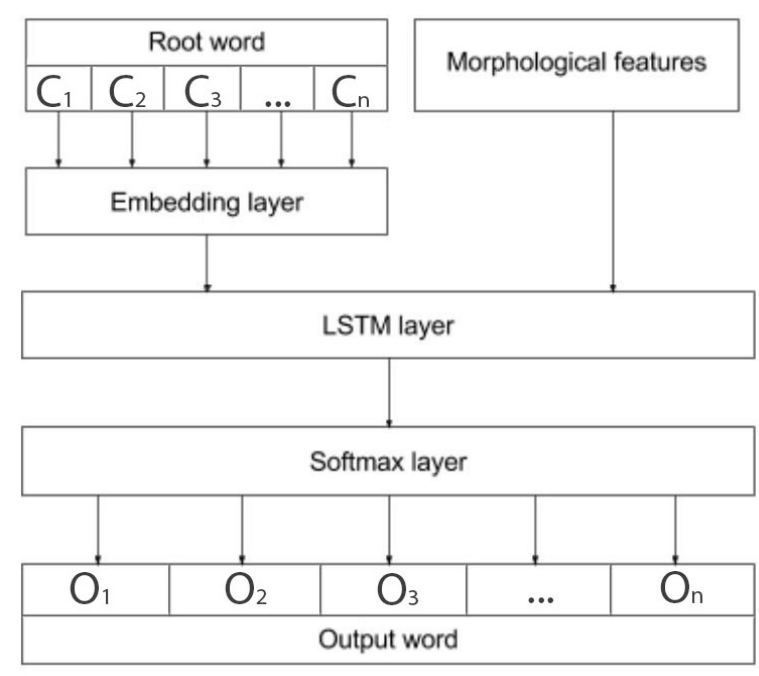

Figure 1: $C_{1}, . ., C_{n}$ represent characters of the root word while $O_{1}, . ., O_{n}$ represent characters of the output word

\subsection{Second Submission}

\subsubsection{Low-sized Dataset}

For training the model on the low-sized dataset, we did not use any encoder and we used a simple GRU, as reported by Cho et al. (2014), with a single layer as the recurrent unit (Figure 3).

\subsubsection{Medium-sized Dataset}

For medium-sized dataset, we used different model configurations for different languages. Four different kinds of configurations were used:

1) Bidirectional LSTM as the encoder and a simple LSTM with a single layer as the decoder (Figure 2) 2) Bidirectional GRU as the encoder and a simple GRU with a single layer as the decoder (Figure 4) 3) No encoder and a simple GRU with a single layer as the recurrent unit (Figure 3) 4) Bidirectional GRU as the encoder and a deep GRU (two GRUs stacked one above the other) as the decoder (Figure 5)

The specific configuration used for each language has been listed in Table 1. The configuration numbers indicated in the table are according to those mentioned above.

\subsubsection{High-sized Dataset}

For high-sized data, we were unable to complete experiments for the second submission due to lack of time. However, we have been able to perform 


\begin{tabular}{|c|c|}
\hline Configuration & Language List \\
\hline 1 & Arabic, Basque, Bengali, Catalan, Georgian, Latin, Quechua, Urdu \\
Kurmanji
\end{tabular}

Table 1: Configurations for different languages for medium-sized data for submission-2.

\begin{tabular}{|l|c|c|c|}
\hline Language & B & S-1(T) & S-2(T) \\
\hline Norwegian-Bokmal & 69.0 & 52.6 & 62.7 \\
Danish & 59.8 & 46.1 & 49.8 \\
Urdu & 30.3 & 31.2 & 43.7 \\
Hindi & 31.0 & 33.4 & 40.8 \\
Swedish & 54.3 & 40.6 & 39.4 \\
\hline
\end{tabular}

Table 2: Accuracies for top-5 languages for low data.

\begin{tabular}{|l|c|c|c|}
\hline Language & BL & S-1 & S-2 \\
\hline Quechua & 68.1 & 93.0 & 93.0 \\
Bengali & 75.0 & 91.0 & 91.0 \\
Portuguese & 92.9 & 86.0 & 89.6 \\
Urdu & 86.1 & 88.0 & 88.0 \\
Georgian & 90.0 & 87.7 & 87.7 \\
\hline
\end{tabular}

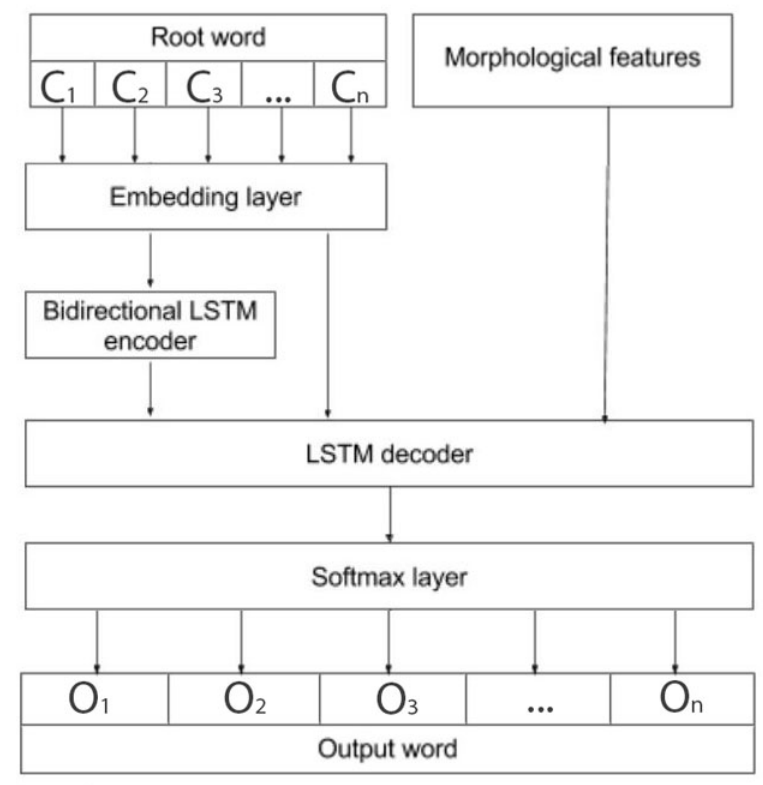

Table 3: Accuracies for top-5 languages for medium data.

\begin{tabular}{|l|c|c|}
\hline Language & BL & S-1 \\
\hline Basque & 6.0 & 100.0 \\
Welsh & 67.0 & 99.4 \\
Hindi & 94.0 & 99.3 \\
Persian & 77.6 & 98.9 \\
Portuguese & 97.4 & 98.5 \\
\hline
\end{tabular}

Table 4: Accuracies for top-5 languages for high data.

\begin{tabular}{|l|c|c|c|}
\hline Language & BL & S-1 & S-2 \\
\hline Norwegian-Bokmal & 0.489 & 0.71 & 0.55 \\
Danish & 0.669 & 0.95 & 0.87 \\
Swedish & 0.884 & 1.08 & 1.09 \\
Norwegian-Nynorsk & 0.928 & 1.41 & 1.23 \\
Dutch & 0.69 & 1.42 & 1.24 \\
\hline
\end{tabular}

Table 5: Levenshtein distances for top-5 languages for low data.

Figure 2: $C_{1}, . ., C_{n}$ represent characters of the root word while $O_{1}, . ., O_{n}$ represent characters of the output word

some ablation studies on high-size datasets, which have been discussed in the analysis section.

\section{Evaluation}

\subsection{Results on Test Set}

The evaluation results were obtained using the evaluation script and the test set provided by the shared task organizers. Baseline accuracies were also obtained from the baseline model provided. The best five baseline accuracies, accuracies for the first submission and accuracies for the second submission can be found in Table 2, Table 3 and Table 4 for each of the three dataset sizes: low, medium and high respectively. Similar results for Levenshtein distances can be found in Table 5, Table 6 and Table 7. In these tables, BL stands for Baseline, S-1 stands for Submission-1 and S-2 stands for Submission-2. 


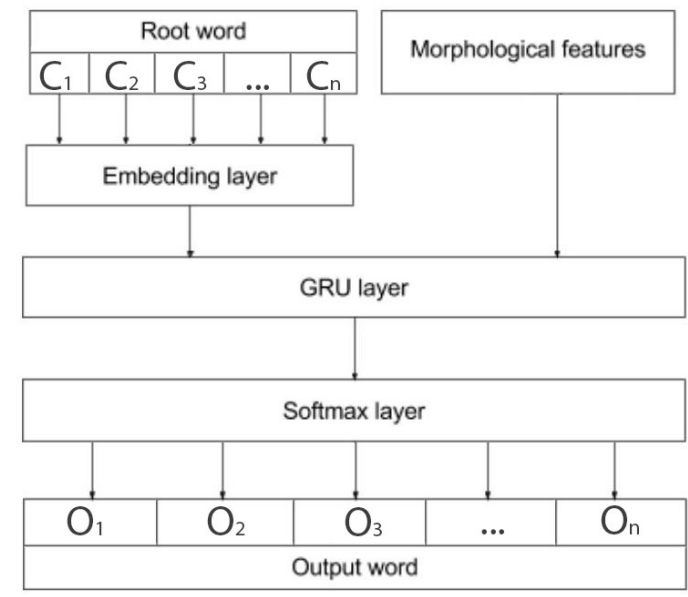

Figure 3: $C_{1}, . ., C_{n}$ represent characters of the root word while $O_{1}, . ., O_{n}$ represent characters of the output word

\begin{tabular}{|l|c|c|c|}
\hline Language & BL & S-1 & S-2 \\
\hline Portuguese & 0.103 & 0.21 & 0.16 \\
Bengali & 0.44 & 0.19 & 0.19 \\
Quechua & 1.706 & 0.28 & 0.28 \\
Welsh & 1.02 & 0.4 & 0.29 \\
Georgian & 0.225 & 0.32 & 0.32 \\
\hline
\end{tabular}

Table 6: Levenshtein distances for top-5 languages for medium data.

The complete set of accuracies and Levenshtein distances for all languages have been included in Appendix-1 (tables 8 to 10), sorted by accuracies. The main observation from these tables is that languages belonging to the same language family tend to get similar similar results by our system, which is intuitively valid (although there are many exceptions). For example, Romance and Slavic languages tend to occur together in these tables.

However, it is not evident from these tables that morphologically more complex languages should be harder to learn, which seems to be counter-

\begin{tabular}{|l|c|c|}
\hline Language & BL & S-1 \\
\hline Basque & 3.32 & 0.0 \\
Serbo-Croatian & 0.36 & 0.0 \\
Welsh & 0.45 & 0.01 \\
Hindi & 0.075 & 0.02 \\
Persian & 0.567 & 0.02 \\
\hline
\end{tabular}

Table 7: Levenshtein distances for top-5 languages for high data.

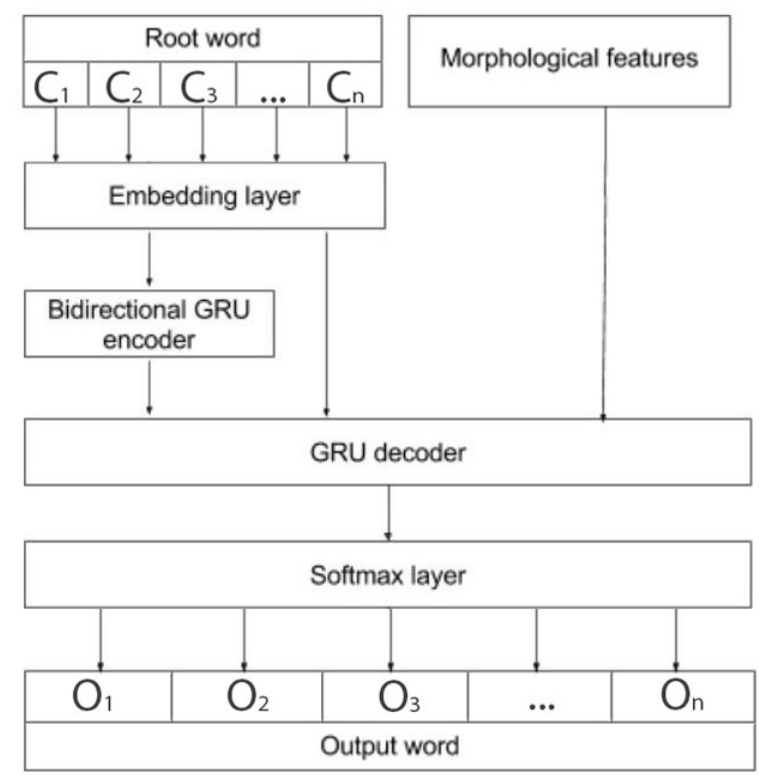

Figure 4: $C_{1}, . ., C_{n}$ represent characters of the root word while $O_{1}, . ., O_{n}$ represent characters of the output word

intuitive. For example, Turkish is above French. This may be because of hyperparameters or configurations selected for different languages (which were different, in an attempt to maximize accuracy on the development data).

Figures 6 to 10 show the correlation between accuracy and Levenshtein distance for all three sizes of datasets for submission- 1 and for low and medium sizes of datasets for submission-2.

\subsection{Ablation Studies}

While we were unable to run an exhaustive hyperparameter search due to lack of time, we performed some experiments, where the choice of hyperparameters was guided by intuitions developed from analysis of the dataset and results obtained on smaller subsets of the data. We have presented some key observations from our analysis in the ensuing sub-sections.

\subsubsection{Early Stop Patience}

We observed that for low-sized datasets, both the models (LSTM as well as GRU based) required that at least 10 epochs be run before early stop, every time no progress is detected on the validation set. Setting this patience to less than 5 , resulted in near 0 accuracies for most languages and printing of nonsensical target words. For medium-sized datasets, this patience value can be set to around 


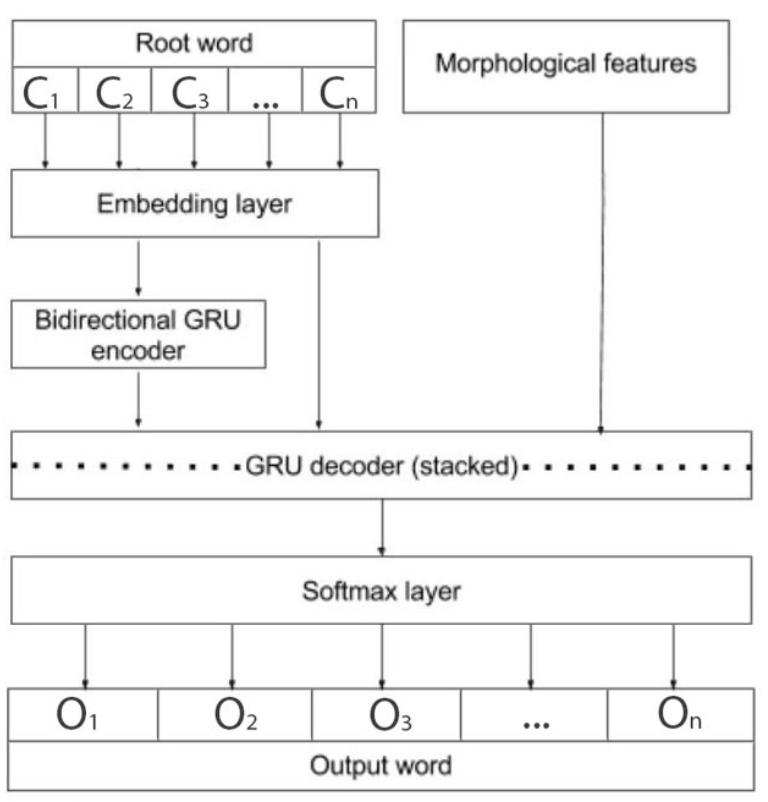

Figure 5: $C_{1}, . ., C_{n}$ represent characters of the root word while $O_{1}, . ., O_{n}$ represent characters of the output word

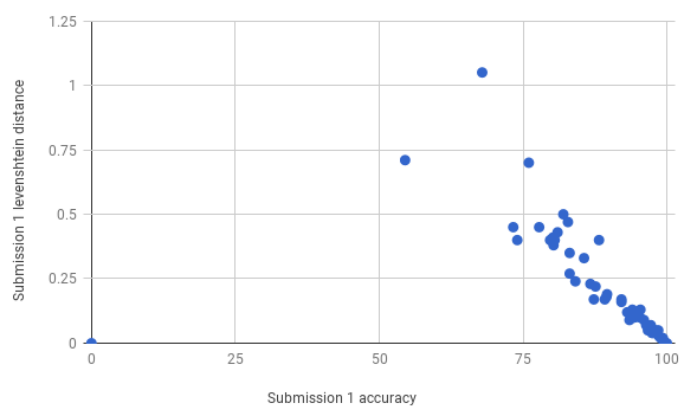

Figure 6: Accuracy vs. Levenshtein Distance for high data (submission-1)

6-8 while for high-sized datasets, it can be set to around 3-4. However, in order to ensure best results, we set our patience value to 10 across all models, training sizes and languages in the final system.

\subsubsection{External Feature Categories}

In last year's version of the shared task, the morphological features in the dataset were annotated along with the category of each feature. For instance, a sample training feature set from last year is: 'pos $=\mathrm{N}, \mathrm{def}=\mathrm{DEF}$,case $=\mathrm{NOM} / \mathrm{ACC} / \mathrm{GEN}, \mathrm{num}=\mathrm{SG}$ '. This year, however, the category of each feature was not provided, i.e., the same example above would appear in this year's format as:

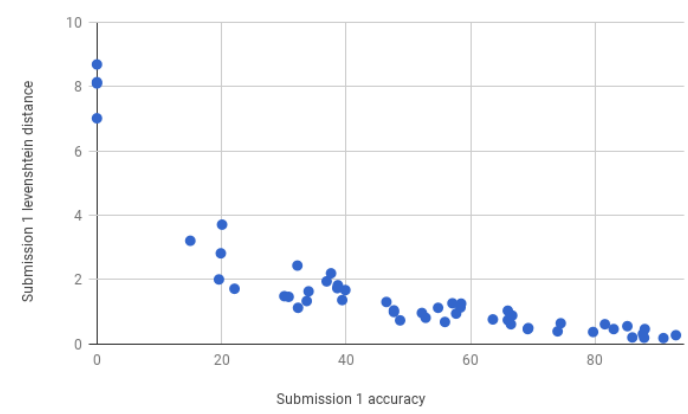

Figure 7: Accuracy vs. Levenshtein Distance for medium data (submission-1)

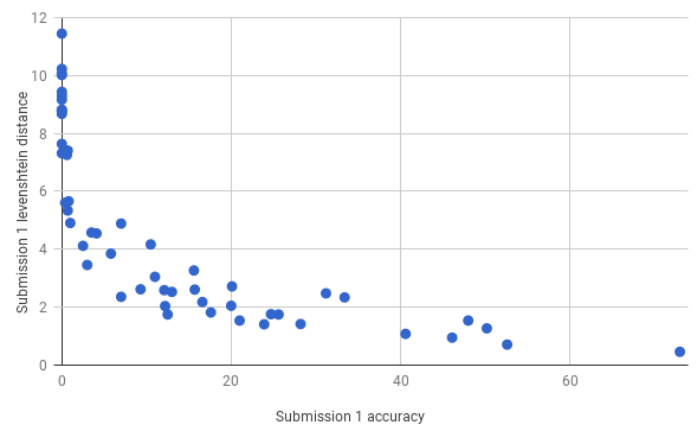

Figure 8: Accuracy vs. Levenshtein Distance for low data (submission-1)

'N,DEF,NOM/ACC/GEN,SG'. Our studies show that while it is conceptually true that the presence of feature categories means exploring a shorter search space, the absence of them does not make a difference to the accuracies obtained for high and medium sized datasets. In the case of low-sized datasets, marginally better accuracies (around 0.5-1\%) were obtained when the categories were incorporated into the dataset (this was done manually). However, this might also be the effect of random initialization of parameters.

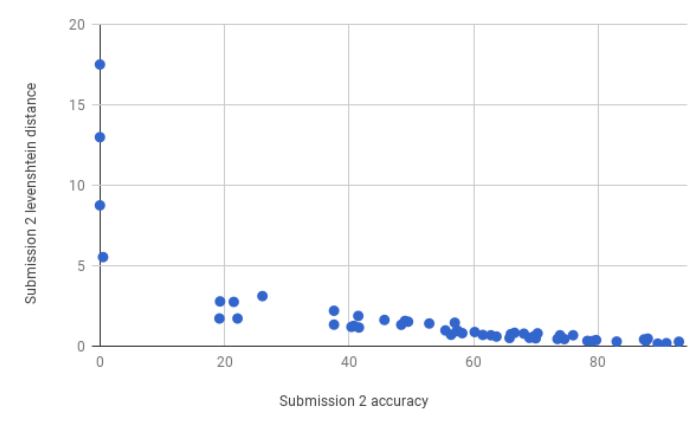

Figure 9: Accuracy vs. Levenshtein Distance for medium data (submission-2) 


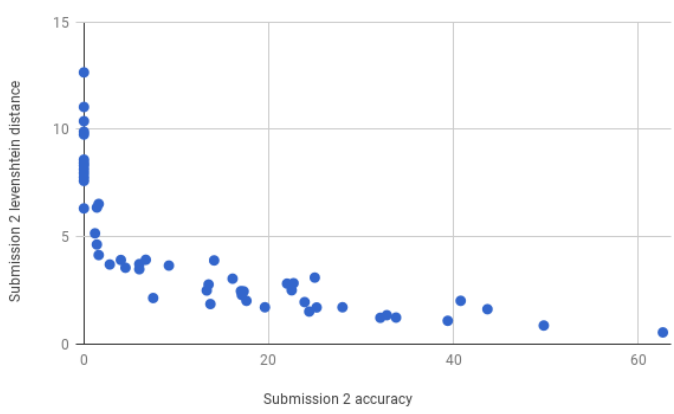

Figure 10: Accuracy vs. Levenshtein Distance for low data (submission-2)

\subsubsection{Choice of Recurrent Unit}

Simple Recurrent Neural Networks (RNNs) performed the poorest on all sizes of datasets. For low-sized datasets, in almost all cases, using a GRU gave better results than using an LSTM. On an average, the accuracy increased by $2.33 \%$ when shifting from LSTM to GRU as the choice of recurrent unit.

In the case of medium-sized datasets, 8 out of 52 languages performed better with an LSTM than a GRU, while the rest showed better performance with a GRU.

\subsubsection{Convolutional Layers}

We also ran experiments using convolutional layers, in which the root word was convolved and the convolution was concatenated along with the root word and passed to the encoder layer (if any). The rest of the network structure remained the same. For low-sized and medium-sized datasets, adding convolutional layers resulted in the accuracy dropping to near 0 . For high-sized datasets, we were unable to finish running the experiments on all languages due to lack of time. However for the few languages on which we performed convolutional ablation studies, it did seem to improve accuracy by around $1.5 \%$ on an average.

\subsubsection{Stacking Recurrent Units}

Deeper models (more than one layer of LSTM/GRU) resulted in drastic accuracy drops for low-sized datasets. For medium-sized datasets, 30 out of 52 languages showed an accuracy improvement upon stacking two GRU layers, while the accuracy drop in the rest 22 was not drastic but appreciable.

\section{Conclusions}

There are two main conclusions. One is that different configurations of deep neural networks work well for different languages. The second is that deep learning may not be the right approach for low-sized data.

Results for low-size were poor for almost all languages. It is to be noted that we used purely deep learning. If deep learning is augmented with other transduction, rule-based or knowledge-based methods, the results for low-size could perhaps be improved.

For high-sized data, for one language (Basque), we even got an accuracy of $100 \%$. For medium, the highest was $93 \%$ and for low, the highest was $69 \%$.

\section{References}

Malin Ahlberg, Markus Forsberg, and Mans Hulden. 2014. Semi-supervised learning of morphological paradigms and lexicons. In Proc. of the 14th Conference of the European Chapter of the Association for Computational Linguistics:Language Technology (Computational Linguistics). Gothenburg, Sweden, pages 569-578.

Malin Ahlberg, Markus Forsberg, and Mans Hulden. 2015. Paradigm classification in supervised learning of morphology. In Proc. of the 2015 Conference of the North American Chapter of the Association for Computational Linguistics: Human Language Technologies. Denver, Colorado, pages 1024-1029.

Kyunghyun Cho, Bart van Merrienboer, Caglar Gulcehre, Fethi Bougares, Holger Schwenk, and Yoshua Bengio. 2014. Learning phrase representations using rnn encoder-decoder for statistical machine translation. In Proc. of EMNLP 2014.

Ryan Cotterell, Christo Kirov, John Walther Géraldine Sylak-Glassman, Ekaterina Vylomova, Patrick Xia, Manaal Faruqui, Sandra Kübler, David Yarowsky, Jason Eisner, and Mans Hulden. 2017. The conllsigmorphon 2016 shared task: Universal morphological reinflection in 52 languages. In Proc. of the CoNLL-SIGMORPHON 2017 Shared Task: Universal Morphological Reinflection. Vancouver, Canada.

Greg Durrett and John DeNero. 2013. Supervised learning of complete morphological paradigms. In Proc. of the 2013 Conference of the North American Chapter of the Association for Computational Linguistics: Human Language Technologies. Atlanta, Georgia, pages 1185-1195.

Manaal Faruqui, Yulia Tsvetkov, Graham Neubig, and Chris Dyer. 2016. Morphological inflection generation using character sequence to sequence learning. In Proc. of NAACL. 
Sepp Hochreiter and Jürgen Schmidhuber. 1997. Long short-term memory. Neural Comput. 9(8):17351780 .

Diederik P. Kingma and Jimmy Ba. 2014. Adam: A method for stochastic optimization. CoRR abs/1412.6980.

\section{Acknowledgement}

We would like to thank Shaili Jain, Aanchal Chaurasia and Himanshu Karu for their help in our experiments in this shared task.

\section{Appendix-1}

In Tables 8 to 10 (on this page and the next), BA stands for baseline accuracy, BLD for baseline Levenshtein Distance, S1A for submission-1 accuracy, S1LD for submission-1 Levenshtein Distance, S2A for submission-2 accuracy and S2LD for submission-2 Levenshtein Distance. All three tables are sorted by submission-1 accuracy, since we have results for all dataset sizes for this submission.

\begin{tabular}{|c|c|c|c|c|}
\hline Language & BA & BLD & S1A & S1D \\
\hline Basque & 6 & 3.32 & 100 & 0 \\
\hline Welsh & 67 & 0.45 & 99.4 & 0.01 \\
\hline Hindi & 94 & 0.075 & 99.3 & 0.02 \\
\hline Persian & 77.6 & 0.567 & 98.9 & 0.02 \\
\hline Portuguese & 97.4 & 0.034 & 98.5 & 0.03 \\
\hline Quechua & 94.7 & 0.106 & 98.5 & 0.05 \\
\hline Bengali & 84 & 0.28 & 98 & 0.05 \\
\hline Georgian & 94 & 0.111 & 97.5 & 0.04 \\
\hline Khaling & 53.8 & 0.816 & 97.4 & 0.04 \\
\hline Catalan & 94.2 & 0.145 & 97.2 & 0.07 \\
\hline Hebrew & 55.8 & 0.551 & 96.7 & 0.05 \\
\hline Ukrainian & 86.3 & 0.289 & 96.4 & 0.07 \\
\hline Haida & 69 & 0.61 & 96 & 0.09 \\
\hline Albanian & 78.1 & 0.606 & 95.4 & 0.13 \\
\hline Italian & 79.9 & 0.624 & 95.3 & 0.1 \\
\hline Estonian & 76.2 & 0.447 & 94.8 & 0.11 \\
\hline Macedonian & 91.9 & 0.152 & 94.6 & 0.12 \\
\hline Bulgarian & 90 & 0.16 & 94.3 & 0.1 \\
\hline English & 95 & 0.09 & 94.3 & 0.1 \\
\hline Sorani & 64.3 & 0.696 & 94.3 & 0.11 \\
\hline Armenian & 89.1 & 0.215 & 94 & 0.12 \\
\hline Swedish & 85.4 & 0.255 & 94 & 0.13 \\
\hline Northern-Sami & 61.1 & 0.813 & 93.6 & 0.12 \\
\hline Kurmanji & 92.2 & 0.088 & 93.5 & 0.09 \\
\hline Lower-Sorbian & 86 & 0.27 & 93.1 & 0.12 \\
\hline Dutch & 86.8 & 0.201 & 92.1 & 0.16 \\
\hline Latvian & 91 & 0.253 & 92.1 & 0.17 \\
\hline Czech & 90.4 & 0.196 & 89.6 & 0.19 \\
\hline Slovene & 89.8 & 0.183 & 89.5 & 0.18 \\
\hline Danish & 89.1 & 0.184 & 89.2 & 0.17 \\
\hline Arabic & 47.7 & 1.481 & 88.2 & 0.4 \\
\hline Urdu & 95.8 & 0.065 & 87.6 & 0.22 \\
\hline Spanish & 90.6 & 0.206 & 87.3 & 0.17 \\
\hline Turkish & 72.9 & 0.772 & 86.7 & 0.23 \\
\hline Navajo & 38.3 & 2.105 & 85.6 & 0.33 \\
\hline Norwegian-Bokmal & 90.6 & 0.154 & 84.1 & 0.24 \\
\hline German & 81.2 & 0.643 & 83.1 & 0.35 \\
\hline Lithuanian & 64.7 & 0.466 & 83.1 & 0.27 \\
\hline Russian & 82 & 0.61 & 82.8 & 0.47 \\
\hline Polish & 89.4 & 0.232 & 82 & 0.5 \\
\hline Slovak & 85.2 & 0.248 & 81 & 0.43 \\
\hline Finnish & 78.5 & 0.361 & 80.5 & 0.4 \\
\hline French & 83.6 & 0.299 & 80.3 & 0.38 \\
\hline Hungarian & 71.1 & 0.622 & 80.2 & 0.41 \\
\hline Icelandic & 76.1 & 0.466 & 79.7 & 0.4 \\
\hline Faroese & 74.7 & 0.553 & 77.8 & 0.45 \\
\hline Romanian & 80.4 & 0.647 & 76 & 0.7 \\
\hline Norwegian-Nynorsk & 78.3 & 0.383 & 73.3 & 0.45 \\
\hline Irish & 54.3 & 1.064 & 67.9 & 1.05 \\
\hline Latin & 45.6 & 0.86 & 54.5 & 0.71 \\
\hline
\end{tabular}

Table 8: Results for all languages for high data, sorted by submission- 1 accuracy 


\begin{tabular}{|c|c|c|c|c|c|c|}
\hline Language & BA & BLD & S1A & S1D & S2A & S2D \\
\hline Quechua & 68.1 & 1.706 & 93 & 0.28 & 93 & 0.28 \\
\hline Bengali & 75 & 0.44 & 91 & 0.19 & 91 & 0.19 \\
\hline Urdu & 86.1 & 0.287 & 88 & 0.47 & 88 & 0.47 \\
\hline English & 90.2 & 0.159 & 87.9 & 0.2 & 0 & 8.74 \\
\hline Georgian & 90 & 0.225 & 87.7 & 0.32 & 87.7 & 0.32 \\
\hline Portuguese & 92.9 & 0.103 & 86 & 0.21 & 89.6 & 0.16 \\
\hline Hindi & 86.6 & 0.186 & 85.2 & 0.56 & 87.4 & 0.42 \\
\hline Haida & 56 & 1.24 & 83 & 0.47 & 0 & 17.48 \\
\hline Kurmanji & 88.4 & 0.234 & 81.6 & 0.62 & 19.2 & 1.72 \\
\hline Catalan & 83.2 & 0.337 & 79.7 & 0.38 & 79.7 & 0.38 \\
\hline Turkish & 33.1 & 2.854 & 74.5 & 0.65 & 0 & 12.97 \\
\hline Welsh & 54 & 1.02 & 74 & 0.4 & 83 & 0.29 \\
\hline Macedonian & 82.3 & 0.323 & 69.3 & 0.5 & 79.1 & 0.32 \\
\hline Danish & 78.1 & 0.336 & 69.2 & 0.47 & 0.5 & 5.53 \\
\hline Spanish & 85.4 & 0.322 & 66.7 & 0.89 & 73.9 & 0.68 \\
\hline Dutch & 71.7 & 0.403 & 66.5 & 0.62 & 74.6 & 0.44 \\
\hline Basque & 2 & 5.11 & 66 & 0.75 & 66 & 0.75 \\
\hline $\begin{array}{l}\text { Scottish- } \\
\text { Gaelic }\end{array}$ & 52 & 0.76 & 66 & 1.04 & 76 & 0.68 \\
\hline French & 76.1 & 0.45 & 63.6 & 0.77 & 69.7 & 0.61 \\
\hline Italian & 73.8 & 0.743 & 58.5 & 1.26 & 70.3 & 0.8 \\
\hline Armenian & 76.6 & 0.442 & 58.4 & 1.14 & 68.1 & 0.78 \\
\hline Latvian & 85.1 & 0.278 & 57.7 & 0.95 & 60.2 & 0.88 \\
\hline Persian & 65.4 & 1.068 & 57.1 & 1.27 & 57 & 1.46 \\
\hline Hebrew & 40 & 0.933 & 55.9 & 0.69 & 65.8 & 0.51 \\
\hline Bulgarian & 75 & 0.445 & 54.8 & 1.13 & 55.5 & 0.98 \\
\hline Slovak & 70.7 & 0.533 & 52.8 & 0.82 & 63.7 & 0.6 \\
\hline Khaling & 18.4 & 1.909 & 52.2 & 0.97 & 58.2 & 0.81 \\
\hline $\begin{array}{c}\text { Norwegian- } \\
\text { Bokmal }\end{array}$ & 79.8 & 0.311 & 48.7 & 0.74 & 78.3 & 0.33 \\
\hline Hungarian & 41.7 & 1.559 & 47.7 & 1.05 & 62.8 & 0.68 \\
\hline Swedish & 73.7 & 0.452 & 47.7 & 1 & 70 & 0.49 \\
\hline Sorani & 52.8 & 1.053 & 46.5 & 1.31 & 57.5 & 0.95 \\
\hline Estonian & 62.4 & 0.779 & 39.9 & 1.68 & 45.7 & 1.63 \\
\hline Russian & 75 & 0.737 & 39.4 & 1.37 & 66.6 & 0.83 \\
\hline Serbo-Croatian & 65.8 & 0.884 & 38.7 & 1.83 & 49.5 & 1.52 \\
\hline Czech & 80.7 & 0.434 & 38.6 & 1.74 & 52.9 & 1.41 \\
\hline Arabic & 40 & 1.787 & 37.6 & 2.2 & 37.6 & 2.2 \\
\hline Romanian & 70.2 & 0.848 & 36.9 & 1.95 & 49 & 1.57 \\
\hline Northern-Sami & 35.7 & 1.445 & 34 & 1.64 & 40.8 & 1.26 \\
\hline Lithuanian & 53 & 0.714 & 33.7 & 1.34 & 37.6 & 1.34 \\
\hline Slovene & 81.9 & 0.33 & 32.3 & 1.13 & 73.5 & 0.45 \\
\hline Albanian & 66.1 & 1.175 & 32.2 & 2.44 & 41.5 & 1.88 \\
\hline Ukrainian & 71.5 & 0.538 & 30.8 & 1.47 & 61.5 & 0.7 \\
\hline German & 71.5 & 0.798 & 30.1 & 1.49 & 57.3 & 0.93 \\
\hline Latin & 36.8 & 1.103 & 22.1 & 1.72 & 22.1 & 1.72 \\
\hline Irish & 44.7 & 1.457 & 20.1 & 3.71 & 26.1 & 3.11 \\
\hline Navajo & 31.3 & 2.495 & 19.9 & 2.82 & 19.3 & 2.78 \\
\hline Polish & 75.2 & 0.533 & 19.6 & 2.01 & 48.4 & 1.33 \\
\hline Finnish & 42.5 & 1.353 & 15 & 3.21 & 21.5 & 2.75 \\
\hline Faroese & 58.7 & 0.891 & 0 & 8.13 & 40.4 & 1.2 \\
\hline Icelandic & 61.4 & 0.763 & 0 & 8.09 & 41.6 & 1.16 \\
\hline Lower-Sorbian & 70.5 & 0.587 & 0 & 7.01 & 69 & 0.52 \\
\hline $\begin{array}{l}\text { Norwegian- } \\
\text { Nynorsk }\end{array}$ & 63.3 & 0.634 & 0 & 8.68 & 56.4 & 0.71 \\
\hline
\end{tabular}

Table 9: Results for all languages for medium data, sorted by submission-1 accuracy

\begin{tabular}{|c|c|c|c|c|c|c|}
\hline Language & $\mathrm{BA}$ & BLD & S1A & S1D & S2A & S2D \\
\hline English & 76.2 & 0.415 & 73 & 0.46 & 0 & 8.14 \\
\hline $\begin{array}{l}\text { Norwegian- } \\
\text { Bokmal }\end{array}$ & 69 & 0.489 & 52.6 & 0.71 & 62.7 & 0.55 \\
\hline Kurmanji & 82.3 & 0.459 & 50.2 & 1.27 & 0 & 7.77 \\
\hline Scottish-Gaelic & 48 & 0.68 & 48 & 1.54 & 0 & 8.32 \\
\hline Danish & 59.8 & 0.669 & 46.1 & 0.95 & 49.8 & 0.87 \\
\hline Swedish & 54.3 & 0.884 & 40.6 & 1.08 & 39.4 & 1.09 \\
\hline Hindi & 31 & 3.798 & 33.4 & 2.34 & 40.8 & 2.02 \\
\hline Urdu & 30.3 & 4.201 & 31.2 & 2.48 & 43.7 & 1.63 \\
\hline Dutch & 53.7 & 0.69 & 28.2 & 1.42 & 33.8 & 1.24 \\
\hline German & 53.7 & 1.111 & 25.6 & 1.75 & 0 & 8.59 \\
\hline Catalan & 55.2 & 1.091 & 24.7 & 1.76 & 25.2 & 1.71 \\
\hline $\begin{array}{l}\text { Norwegian- } \\
\text { Nynorsk }\end{array}$ & 50.8 & 0.928 & 23.9 & 1.41 & 32.1 & 1.23 \\
\hline Slovene & 47.4 & 0.862 & 21 & 1.54 & 0 & 7.6 \\
\hline Spanish & 58.6 & 1.229 & 20.1 & 2.72 & 22.5 & 2.51 \\
\hline Bengali & 44 & 1.49 & 20 & 2.05 & 28 & 1.72 \\
\hline Lower-Sorbian & 34.3 & 1.264 & 17.6 & 1.82 & 19.6 & 1.72 \\
\hline Latvian & 62.1 & 0.806 & 16.6 & 2.18 & 17.6 & 2.02 \\
\hline Russian & 42.8 & 1.311 & 15.7 & 2.61 & 17.3 & 2.46 \\
\hline Czech & 40.8 & 1.869 & 15.6 & 3.27 & 16.1 & 3.05 \\
\hline Icelandic & 34.2 & 1.541 & 13 & 2.53 & 13.3 & 2.5 \\
\hline Slovak & 41.9 & 1.029 & 12.5 & 1.75 & 0 & 6.32 \\
\hline Ukrainian & 40.7 & 1.001 & 12.2 & 2.04 & 13.7 & 1.87 \\
\hline Polish & 41.9 & 1.551 & 12.1 & 2.59 & 17.1 & 2.29 \\
\hline Bulgarian & 33.1 & 1.572 & 11 & 3.05 & 13.5 & 2.78 \\
\hline Persian & 27.3 & 3.357 & 10.5 & 4.17 & 14.1 & 3.9 \\
\hline Faroese & 30.7 & 1.585 & 9.3 & 2.62 & 4.5 & 3.56 \\
\hline Haida & 34 & 6.03 & 7 & 4.89 & 25 & 3.1 \\
\hline Hebrew & 27.9 & 1.312 & 7 & 2.36 & 7.5 & 2.15 \\
\hline Romanian & 44.1 & 1.551 & 5.8 & 3.85 & 1.6 & 4.15 \\
\hline $\begin{array}{l}\text { Serbo- } \\
\text { Croatian }\end{array}$ & 21.3 & 2.735 & 4.1 & 4.55 & 9.2 & 3.66 \\
\hline Estonian & 22.6 & 2.93 & 3.5 & 4.58 & 6.7 & 3.93 \\
\hline Lithuanian & 23.5 & 1.916 & 3 & 3.46 & 0 & 8.44 \\
\hline Northern-Sami & 15.4 & 2.359 & 2.5 & 4.12 & 4 & 3.92 \\
\hline Basque & 0 & 6.46 & 1 & 4.91 & 6 & 3.73 \\
\hline Arabic & 21.5 & 3.049 & 0.8 & 5.66 & 0 & 9.76 \\
\hline Quechua & 17.2 & 6.691 & 0.7 & 5.34 & 22.7 & 2.84 \\
\hline Finnish & 16.2 & 4.217 & 0.7 & 7.41 & 1.6 & 6.53 \\
\hline Irish & 31.8 & 2.698 & 0.6 & 7.26 & 0 & 9.89 \\
\hline Navajo & 18.4 & 3.432 & 0.4 & 5.61 & 1.2 & 5.16 \\
\hline Portuguese & 60.3 & 0.956 & 0 & 9.31 & 32.8 & 1.35 \\
\hline Macedonian & 50 & 1.006 & 0 & 8.68 & 24.4 & 1.52 \\
\hline French & 63 & 0.781 & 0 & 8.8 & 23.9 & 1.96 \\
\hline Armenian & 37.8 & 2.218 & 0 & 9.17 & 22 & 2.82 \\
\hline Welsh & 15 & 1.6 & 0 & 8.77 & 17 & 2.47 \\
\hline Latin & 16 & 2.838 & 0 & 9.44 & 6 & 3.49 \\
\hline Khaling & 3.9 & 4.298 & 0 & 7.32 & 2.8 & 3.71 \\
\hline Albanian & 21.6 & 4.439 & 0 & 10.23 & 1.4 & 6.36 \\
\hline Sorani & 20.5 & 3.363 & 0 & 7.64 & 1.4 & 4.64 \\
\hline Georgian & 71.2 & 0.585 & 0 & 8.82 & 0 & 7.96 \\
\hline Hungarian & 17.2 & 2.049 & 0 & 10.07 & 0 & 11.04 \\
\hline Italian & 44.9 & 1.998 & 0 & 10.02 & 0 & 10.38 \\
\hline Turkish & 14.3 & 4.319 & 0 & 11.45 & 0 & 12.65 \\
\hline
\end{tabular}

Table 10: Results for all languages for low data, sorted by submission- 1 accuracy 